\title{
Chandra Observations of Clusters of Galaxies
}

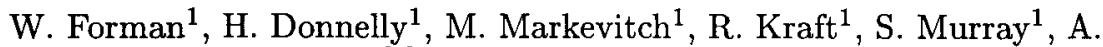 \\ Vikhlinin $^{2}$, E. Churazov ${ }^{3,2}$, L. David ${ }^{1} \&$ C. Jones ${ }^{1}$ \\ 1 - Harvard-Smithsonian Center for Astrophysics, 60 Garden St. \\ Cambridge, $M A 02138$ \\ 2 - Institute for Space Research, Profsouznaya 84/32, 117810 Moscow, \\ Russia \\ 3 - MPI fur Astrophysik, Karl-Schwartzschild Strasse 1, 85740 \\ Garching, Germany
}

\begin{abstract}
We discuss two themes from Chandra cluster observations. First, we describe the interaction of buoyant, radio emitting plasma bubbles with the hot intracluster gas. Second we summarize the Chandra observations of "cold" fronts (sharp discontinuities in gas density and temperature) separating cool, denser gas clouds from the hotter intracluster medium.
\end{abstract}

\section{The Radio - X-ray Interaction or Bubbles, Bubbles Everywhere}

Prior to the launch of Chandra, ROSAT observations of NGC1275 and M87 provided hints of complex interactions between radio emitting plasmas ejected from central galaxies in clusters (e.g., Böhringer et al. 1993, 1995; Churazov et al. 2000a).

The prevalence of the interaction between the radio emitting plasma and the hot intracluster medium (ICM) has increased dramatically with the launch of Chandra. In the Hydra A cluster, Chandra observations showed cavities created by the inner radio lobes as they displaced the X-ray gas (McNamara et al. 2000). In addition, a complex X-ray enhancement is associated with the northern buoyant radio bubble (Taylor et al. 1990). For NGC1275/Perseus, Fabian et al. (2000) showed that the bright edges around the X-ray holes (radio lobes) were not produced by shocks since their emission was softer than that of the ambient hot gas. Finoguenov \& Jones (2000) found a very complex structure for the X-ray emitting gas around the Virgo galaxy M84 (NGC4374) that is determined by the morphology of the radio lobes. The ACIS Centaurus A image shows an X-ray enhancement just in advance of the leading edge of the southern inner radio lobe (see Kraft et al. 2000 for a discussion of the HRC image).

In a study of M87, Churazov et al. (2000b) combined the new radio image of Owen, Eilek, \& Kassim (2000) with the ROSAT HRI data to describe the interaction of buoyant bubbles with the X-ray emitting hot gas and to explain many features seen in clusters and around galaxies as the radio plasma interacts with the hot gas. They liken the behavior of the bubbles to that of atmospheric 

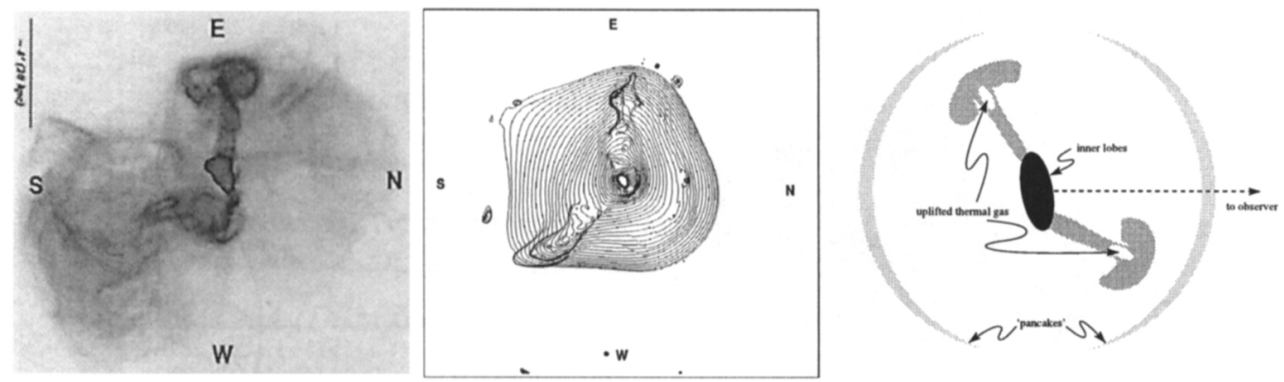

Figure 1. Left: $14^{\prime} .6 \times 16^{\prime} .0$ radio map of M87 (North right, East up) (from Owen et al. 2000). Center: Contour map of the smoothed ROSAT HRI X-ray image (same size and orientation as the radio image). Right: Suggested source geometry. The central black region denotes the inner radio lobes, the gray "mushrooms" correspond to buoyant bubbles already transformed into tori, and the gray lens-shaped structures are "pancakes" (seen edge-on) formed by older bubbles.

nuclear explosions in which the bubbles are inflated and then rise under their own buoyancy. During their rise, the bubbles transform into toroidal structures and uplift cooler gas from lower in the cluster atmosphere. The rising tori may be trailed by columns of cool, dense (and hence bright) gas. Finally, when reaching that height at which the bubble's mean density (including any entrained gas) equals the ambient density, the bubble spreads laterally along equipotential surfaces to form a thin pancake-like "sheet". Fig. 1 illustrates the appearance of bubbles at different evolutionary phases in M87.

\section{Cluster Mergers - into the Cauldron}

ROSAT and ASCA provided a tantalizing view of the rich physics that might be expected from detailed studies of cluster mergers (e.g., Henriksen \& Markevitch 1996; Churazov et al. 1999; Henriksen, Donnelly, \& Davis 2000). One particular feature of cluster mergers was a sharp "edge" seen in A3667 that was suggested to be a shock (Markevitch, Sarazin, \& Vikhlinin 1999). The first Chandra observations of edges in A2142 (Markevitch et al. 2000) and A3667 (Vikhlinin, Murray, \& Markevitch 2000a, b) have shown that these features arise, not from shocks, but from cold clouds traversing the ICM.

The deep A3667 Chandra observations show that the temperature and density discontinuities occur over less than $5 \mathrm{kpc}\left(3.5^{\prime \prime}\right)$, smaller than the particle mean free paths, thus requiring the suppression of transport processes (see Fig. 2). The front is not a shock since the denser region is also cooler. Furthermore, to suppress the Kelvin-Helmholtz instability, pressure equilibrium across the front requires that the cold cloud move at nearly the sonic velocity. Finally, the sharpness of the discontinuity, its extent, and the tangential gas velocity around the moving front requires a magnetic field with $P_{\text {mag }} / P_{\text {gas }} \sim 0.1-0.2$.

Acknowledgments. We acknowledge support from NASA contract NAS8 39073, NASA grants NAG5-3065 and NAG5-6749 and the Smithsonian Institution. 

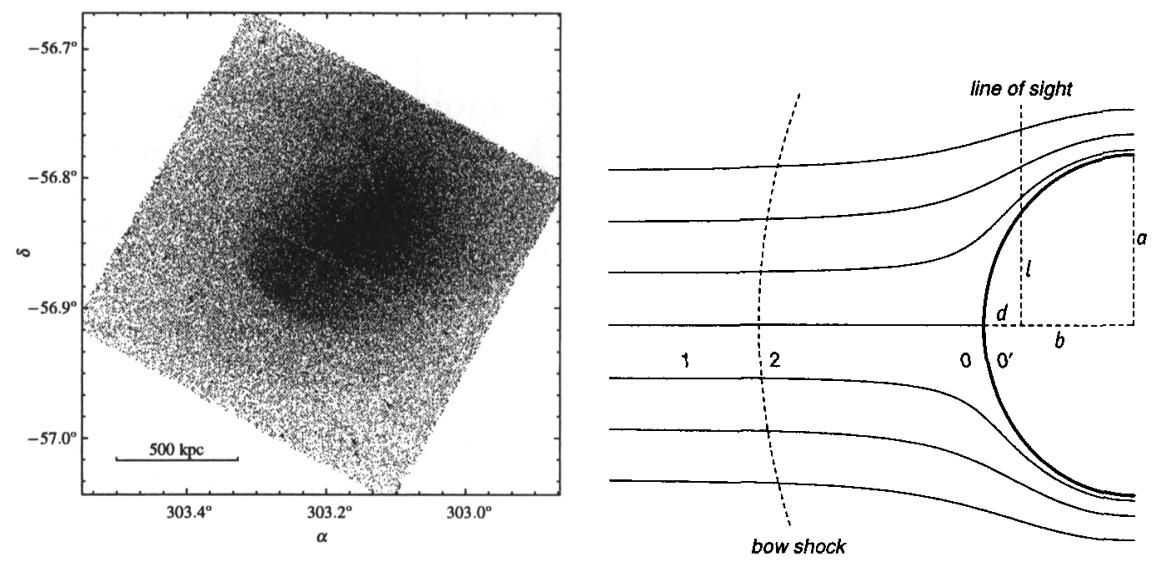

Figure 2. Left: Chandra image of A3667 showing the sharp surface brightness discontinuity to the south south-west. Right: Geometry of flow past a spheroid (semi-axes $a$ and $b$ ). Zones $0,1,2$ are those near the stagnation point, in the undisturbed free stream, and just past a possible bow shock (weakly seen in the X-ray image), respectively.

\section{References}

Böhringer, H. et al. 1993, MNRAS, 264, L25

Böhringer, H., Nulsen, P., Braun, R., Fabian, A. 1995, MNRAS, 274, L67

Churazov, E. et al. 1999, ApJ, 520, 105

Churazov, E. et al. 2000a, A\&A, 356, 788

Churazov, E. et al. 2000b, submitted to ApJ (astro-ph/0008215)

Fabian, A. et al. 2000, MNRAS in press (astro-ph/0007456)

Finoguenov, A., Jones, C. 2000, submitted to ApJ

Henriksen, M., Donnelly, H., Davis, D. 2000, ApJ, 529, 692

Henriksen, M. \& Markevitch, M. 1996, ApJL, 466, 79

Kraft, R. et al. 2000, ApJL, 531, 9

Markevitch, M., Sarazin, C., Vikhlinin, A. 1999, ApJ, 521, 526

Markevitch, M. et al. 2000, ApJ in press (astro-ph/0001269)

McNamara, B. et al. 2000, ApJL, 534, 135

Owen, F., Eilek, J, Kassim, N. 2000, ApJ in press (astro-ph/0006150)

Taylor, G. et al. 1990, ApJ, 360, 41

Vikhlinin, A., Markevitch, M., Murray, S. 2000a, ApJ submitted (astro$\mathrm{ph} / 0008496)$

Vikhlinin, A., Markevitch, M., Murray, S. 2000b, ApJ submitted (astro$\mathrm{ph} / 0008499)$ 